\title{
Human Papilloma Virus Vaccine: Future of Cervical Cancer Prevention
}

\author{
Jannatul Fardows ${ }^{1}$, Naznin Nehar ${ }^{2}$, Nurjahan Laskar ${ }^{3}$, Samsoon Nahar Joly ${ }^{4}$ \\ Received: December 15, 2015 Accepted: August 31, 2016 \\ doi: http://dx.doi.org/10.3329/jemc.v6i3.29683
}

\begin{abstract}
Cervical cancer is a deadly cancer that clutches lives of the women in most of the cases due to lack of consciousness about the disease in the developing countries. It remains a threat which is second only to breast cancer in overall disease burden for women throughout the world. Cervical cancer is almost a preventable disease by prophylactic vaccine and routine screening. Both Cervarix and Gardasil vaccines have been effective in preventing persistent infection with targeted HPV types and in preventing cervical intraepithelial lesions. It is safe and nearly $100 \%$ effective if given before onset of sexual activity. This review article is aimed to explore different aspects of this vaccine as well as to develop awareness among health professionals of different disciplines.
\end{abstract}

Key words: Human papilloma virus; Cervical cancer; Vaccine; Cervarix; Gardasil; Efficacy

J Enam Med Col 2016; 6(3): 157-160

\section{Introduction}

Cervical cancer remains a critical public health problem that is second only to breast cancer in overall disease burden for women throughout the world. ${ }^{1}$ It is still an important cause of mortality and morbidity in the developing countries. ${ }^{2}$ Universal deployment of organized or opportunistic screening with Pap cytology in high and middle income countries has been the primary reason for the substantial reductions in cervical cancer morbidity and mortality during the last 50 years. However, in many low income countries Pap cytology screening is yet to be effectively implemented or has failed to reduce cervical cancer rates to an appreciable extent. $^{3}$ Screening appears to benefit only a small fraction of women although a much larger percentage endures the inconvenience of the Pap test in order to avoid cervical cancer. ${ }^{2}$

Human papilloma virus (HPV) infection is one of the most common sexually transmitted diseases worldwide. Up to 79 million of sexually active women worldwide will become infected with HPV during their lifetime. ${ }^{4}$ To date, more than 120 genotypes of Human Papilloma virus (HPV) are known, but only high risk oncogen genotypes could induce a cancer. HPV 16 and 18 are implied in nearly $70 \%$ of cervical cancer around the world. ${ }^{5}$ In Bangladesh, the annual number of cases of cervical cancer is 11956 and death is $6582 .{ }^{6} \mathrm{~A}$ prophylactic vaccine to protect against the precancerous and cancerous lesions associated with HPV should save lives, reduce expensive diagnostic and therapeutic interventions, and have substantial individual and collective benefits. ${ }^{5}$ In this review paper, we discuss the type of cervical vaccine and its mechanism of action. Various reports of trial and vaccine impact on different categories of women are also discussed.

\section{Function of vaccine}

The current HPV vaccines are based on virus-like particles (VLPs) that are formed by HPV surface components (viral capsid). They are not infectious, because they lack the virus' DNA. However, they

1. Assistant Professor, Department of Microbiology, International Medical College, Gazipur

2. Lecturer, Department of Virology, Dhaka Medical College, Dhaka

3. Assistant Professor, Department of Microbiology, International Medical College, Gazipur

4. Assistant Professor, Department of Microbiology, Kumudini Women's Medical College, Tangail

Correspondence Jannatul Fardows, Email: jannat2026@gmail.com 
closely resemble the natural virus, and antibodies against the VLPs also have activity against the natural virus. The VLPs have been found to be strongly immunogenic, which means that they induce high levels of antibody production by the body. ${ }^{7}$ The antibody induces more than $90 \%$ protection from persistent HPV 16, 18 infection and HPV 16 and 18 associated high grades cervical intraepithelial neoplasia. ${ }^{8} \mathrm{HPV} 16$ and 18 vaccination will prevent HPV 16, 18 infection, and subsequently decreases in $90 \%$ the frequency of abnormal Pap attributable to these types. HPV vaccination will reduce the number of women who require colposcopy, biopsy and cervical treatment for precancerous cervical lesions. ${ }^{1}$

\section{Types of vaccine}

Three vaccines are approved by the FDA to prevent HPV infection: Gardasil, Gardasil 9, and Cervarix. All three vaccines prevent infections with HPV types 16 and 18, two high-risk HPVs that cause about $70 \%$ of cervical cancers and an even higher percentage of some of the other HPV-associated cancers. ${ }^{9}$ Gardasil also prevents infection with HPV types 6 and 11 which cause $90 \%$ of genital warts. ${ }^{10}$ Because Gardasil protects against infection with four HPV types, it is called a quadrivalent vaccine. Gardasil 9 prevents infection with the same four HPV types plus five additional high-risk HPV types $(31,33,45,52$, and 58) and is therefore called a nonavalent, or 9-valent vaccine. All three vaccines are given through a series of three injections into muscle tissue over a six months period ( 0 month, 1 month and 6 month or 0 month, 2 month and 6 month) and the need for boosters is unknown. ${ }^{11}$ The best age for primary vaccination appears to be $11-12$ or 14 years, before the onset of sexual activity. ${ }^{12}$ The FDA has approved Gardasil and Gardasil 9 for use in females aged 9 through 26 for the prevention of HPV-caused cervical, vulvar, vaginal and anal cancers, precancerous cervical lesions and genital warts. Gardasil and Gardasil 9 are also approved for use in males for the prevention of HPV-caused anal cancer, precancerous anal lesions and genital warts. ${ }^{13}$ It targets two HPV types - 16 and 18 and is called a bivalent vaccine. The FDA has approved Cervarix for use in females aged 9 through 25 for the prevention of cervical cancer caused by HPV. Both vaccines have a known duration of protection of at least 5 years; clinical trials are still in progress to show the full duration of protection. ${ }^{13}$

\section{Side effects}

Side effects include pain, redness or swelling at the injection site, mild fever and itching at the injection site. However, these symptoms do not last long and life-threatening allergic reactions from vaccines are very rare. $^{14}$

\section{Vaccination in special situations}

\section{Women with previous cervical intraepithelial neoplasia (CIN)}

The vaccine can be given to patients with previous CIN, but the benefits may be limited to the protection against infection of HPV genotypes (and related CIN) with which they have not been infected and this vaccine is not for treatment. It must be emphasized that cervical cytology surveillance and corresponding management must continue. $^{15}$

\section{Pregnancy and lactating women}

The use of the vaccine in pregnancy is not recommen-ded although no teratogenic effect caused by the vaccine has been reported. There is no evidence to show that the HPV vaccine adversely affects fertility, pregnancy or infant outcome. ${ }^{16}$

- Women who are planning to conceive are advised to defer vaccination until after delivery.

- Women who become pregnant before completion of vaccination are advised to postpone the remaining dose until after the pregnancy.

- Termination of pregnancy is not indicated for women who become inadvertently pregnant during the course of vaccination.

- Lactating women can receive the HPV vaccine and still continue breastfeeding because it is a vaccine without live viral DNA.

\section{Immunosuppressed patients}

Immunosuppression is not a contraindication to vaccination. However, the immune response to the HPV vaccine may be less competent in these women compared with a healthy individual. ${ }^{17}$

\section{Contraindications and precautions}

The HPV vaccine is contraindicated for people with a history of hypersensitivity to any vaccine component. Vaccination of people with moderate or severe acute 
illnesses should be deferred until after the illness improves. ${ }^{16}$

\section{Cervical cancer and vaccine status in developing countries}

Cervical cancer is the most prevalent cancer that continues to be a major health care problem worldwide and it is still an important and common gynecological cancer in the developing countries. Cervical cancer constitutes about 21-23\% of the female cancer in different areas of India and Bangladesh. ${ }^{2}$ Bangladesh stands $11^{\text {th }}$ for cervical cancer fatalities in the world. Data collected from different hospitals reveal that cervical cancer is the commonest cancer in Bangladesh. ${ }^{18}$ Prevalence of cervical cancer is also high in other developing countries like Senegal, Madagascar, Malawi, Nigeria and Sierra Leone. This high prevalence of cervical cancer is related to many risk factors as early marriage, early starting of sexual activity, multiparity, low socioeconomic condition and high incidence of sexually transmitted diseases and HPV infection. ${ }^{19}$

Not only the different risk factors for cervical cancer but also the price of vaccine is the other main factor. The retail price of the vaccines is approximately $\$ 130$ to $\$ 160$ per dose worldwide. ${ }^{20}$ It is really impossible for low income countries to afford this price. But according to Global Alliance for Vaccines and Immunization (GAVI), more than $85 \%$ of the 275,000 global deaths every year due to cervical cancer are in low-income nations. The HPV vaccine is available for routine immunization in relatively wealthy countries until now. But recently, GAVI has announced that they have already outlined plans to introduce HPV vaccine in routine immunization program with record low price for the low income countries $-\$ 4.5$ per dose. ${ }^{20}$ In Bangladesh, Cervarix vaccine is available and manufactured by GlaxoSmithKline (GSK). It contains two HPV types - 16 and 18.

\section{Vaccine efficacy}

HPV vaccines are highly effective in preventing infection with the types of HPV they target when given before initial exposure to the virus, which means before onset of sexual activity. In a combined analysis of the initial efficacy and extended follow-up studies, vaccine efficacy of $100 \%$ against cervical intraepithelial neoplasia (CIN) lesions is associated with vaccine types. ${ }^{4}$ In another study that led to approval of Gardasil and Cervarix, these vaccines were found to provide nearly $100 \%$ protection against persistent cervical infections with HPV types 16 and 18 and the cervical cell changes that these persistent infections can cause. ${ }^{21}$ A clinical trial of Gardasil in men indicated that it can prevent anal cell changes caused by persistent infection and genital warts. ${ }^{22}$ Although these two vaccines give 5-year protection, long-term studies of vaccine efficacy that are still in progress will help scientists better understand the total duration of protection.

\section{Conclusion}

In conclusion, cancer cervix is a potentially preventable disease. But due to lack of proper knowledge about disease and vaccine, it is still a major health problem and burden to the developing countries. HPV vaccination of girls before sexual debut could be a potential tool for the prevention and control of cervical cancer. According to GAVI, if it is possible to introduce vaccine in routine immunization program with low price in low income countries and if proper screening is done at the precancerous stage, in future the rate of cervical cancer will decrease to a greater extent.

\section{Acknowledgement}

We thank Dr. Maksud Mazumder, Registrar, Department of Medicine, Popular Medical College, Dhaka and Dr. Abu Bakar Siddique, Assistant Registrar, Department of Medicine, Dhaka Medical College, Dhaka, for their contribution to prepare this review paper.

\section{References}

1. Harash KH, Tanyas S. Study on knowledge and screening for cervical cancer among women in Mangalore city, India. Ann Med Sci Res 2014; 4(5): 751-756.

2. Sultana R, Sultana N. Clinical profile and treatment protocol of invasive carcinoma of cervix. Bang Med J 2012; 45: 11-14.

3. Bamanikar SA, Baravkar DS, Chandanwale SS, Depkekar P. Study of cervical pap smears in a tertiary hospital. Indian Med Gazette 2014: 250-254.

4. CDC (Centers for Disease Control and Prevention). Epidemiology and prevention of vaccine-preventable diseases. CDC, Atlanta, 2015. Available at: www.cdc.gov/vaccine/pubs/ pinkbook/. Accessed July 2016.

5. CDC (Centers for Disease Control and Prevention). Human papilloma vaccination. Recommendation of the Advisory Committee on Immunization Practice (ACIP). Morb Mortal Wkly Rep 2014; 63 (5): 1-30. 
6. Brumil L, Albero G, Aldea M, Serrano B, Mena M, Cosano $\mathrm{R}$ et al. Human papilloma virus and related diseases in Bangladesh. Summary report, 2016. Available at: www. hpvcentre.net>reports>. Accessed July 2016.

7. Morshed K, Polz-Gruszka D. Human papillomavirus structure, epidemiology and pathogenesis. J Otolaryngol 2014; 65(5): 213-219.

8. Stanley MA. Human papillomavirus vaccines. Rev Med Virol 2012; V16(3): 139-149.

9. Vincenzo RD, Conte C, Ricci C, Scambia G, Capellia G. Long-term efficacy and safety of human papilloma virus vaccination. Int J Women's Health 2014; 6: 999-1010.

10. CDC (Centers for Disease Control and Prevention). Human papilloma virus vaccine information for clinicians. Fact sheet, 2015. Available at: www.cdc.gov/fact sheet/. Accessed July 2016.

11. Ferris D, Samakoses R, Block SL. Long-term study of a quadrivalent human papillomavirus vaccine. J Pediatri 2014; 134(3): 657-665.

12. CDC (Centers for Disease Control and Prevention). Human papilloma virus. Fact sheet, 2012. Available at: www.cdc. gov/fact sheet/. Accessed July 2016.

13. Collins S, Mazloomzadeh S, Winter H. High incidence of cervical human papillomavirus infection in women during their first sexual relationship. British J Obs Gynaecol 2012; 109(1): 96-98.

14. Gee J, Naleway A, Shui I. Monitoring the safety of quadrivalent human papillomavirus vaccine: findings from the vaccine safety datalink. Vaccine 2011; 29(46): 8279-8284.

15. Siddiqui M. Cervical cancer vaccination: a new hope. AKMMC J 2011; 2(1): 26-31.
16. CDC (Centers for Disease Control and Prevention). FDA licensure of bivalent human papillomavirus vaccine (HPV2, Cervarix) for use in females and updated HPV vaccination recommendations from the Advisory Committee on Immunization Practices (ACIP). Morb Mortal Wkly Rep 2013; 59(20): 626-629.

17. Petrosky E, Bocchini JA, Jr., Hariri S. Use of 9-Valent Human Papillomavirus (HPV) Vaccine: updated HPV vaccination recommendations of the Advisory Committee on Immunization Practices. Morbidity and Mortality Weekly Report. 2015; 64(11): 300-304.

18. Annual report of National Institute of Cancer Research and Hospital, Bangladesh, 2015. Available at: bdnews24.com> health. Accessed July 2016.

19. Reis N, Beji NK, Kilic D. Risk factors for cervical cancer: result from a hospital based case-control study. Int $\mathrm{J}$ Hematol Oncol 2015; 3(21): 153-159.

20. CDC (Centers for Disease Control and Prevention). CDC vaccine price list, as of April 1, 2015. Retrieved April 28, 2015. Available at: http://www.cdc.gov/vaccines/programs/ vfc/awardees/vaccine-management/price-list/. Accessed July 2016.

21. Khatun S, Hussain SMK, Chowdhury S, Ferdous J, Hossain F, Begum SR. Safety and immunogenicity profile of Human Papillomavirus-16/18, AS04 Adjuvant cervical cancer vaccine: a randomized controlled trial in healthy adolescent girls of Bangladesh. Jpn J Clin Oncol 2012; 42(1): 36-41.

22. Giuliano AR, Palefsky JM, Goldstone S. Efficacy of quadrivalent HPV vaccine against HPV Infection and disease in males. New England J Med 2011; 364(5): 401-411. 